Algebraic 83 Geometric $\mathcal{T}_{\text {opology }}$

Volume 5 (2005) 31-51

Published: 7 January 2005

ATG

\title{
On Davis-Januszkiewicz homotopy types I; formality and rationalisation
}

\author{
DiETRICH NotBOHM \\ Nigel RAY
}

\begin{abstract}
For an arbitrary simplicial complex $K$, Davis and Januszkiewicz have defined a family of homotopy equivalent $\mathrm{CW}$-complexes whose integral cohomology rings are isomorphic to the Stanley-Reisner algebra of $K$. Subsequently, Buchstaber and Panov gave an alternative construction (here called $c(K)$ ), which they showed to be homotopy equivalent to Davis and Januszkiewicz's examples. It is therefore natural to investigate the extent to which the homotopy type of a space is determined by having such a cohomology ring. We begin this study here, in the context of model category theory. In particular, we extend work of Franz by showing that the singular cochain algebra of $c(K)$ is formal as a differential graded noncommutative algebra. We specialise to the rationals by proving the corresponding result for Sullivan's commutative cochain algebra, and deduce that the rationalisation of $c(K)$ is unique for a special family of complexes $K$. In a sequel, we will consider the uniqueness of $c(K)$ at each prime separately, and apply Sullivan's arithmetic square to produce global results for this family.
\end{abstract}

AMS Classification 55P62, 55U05; 05E99

Keywords Colimit, formality, Davis-Januszkiewicz space, homotopy colimit, model category, rationalisation, Stanley-Reisner algebra

\section{Introduction}

Over the last decade, work of Davis and Januszkiewicz [7 has popularised homotopy theoretical aspects of toric geometry amongst algebraic topologists. The results of [7] have been surveyed by Buchstaber and Panov in [4, where several further applications were developed. Their constructions have led us to consider the uniqueness of certain associated homotopy types, and our aim is to begin that study here; we focus on general issues of formality, and deduce that the rationalisations of a family of special cases are unique. In a sequel [19, we discuss the problem prime by prime, and obtain global results for members 
of the family by appeal to Sullivan's arithmetic square. The general problem of uniqueness appears to be of considerable difficulty.

We work over an arbitrary commutative ring $R$ with identity, and consider a universal set $V$ of vertices $v_{1}, \ldots, v_{m}$, ordered by their subscripts. The vertices masquerade as algebraically independent variables, which generate a graded polynomial algebra $S_{R}(V)$ over $R$. The grading is defined by assigning each of the generators a common dimension, which we usually take to be 2 . A function $M: V \rightarrow \mathbb{N}$ is known as a multiset on $V$, with cardinality $|M|:=\sum_{j} M\left(v_{j}\right) ;$ it may be represented by the monomial $v_{M}:=\prod_{V} v^{M(v)}$, or by the $n$-tuple of constituent vertices $\left(v_{j_{1}}, \ldots, v_{j_{n}}\right)$, where $j_{1} \leq \cdots \leq j_{n}$ and $n=|M|$. So $S_{R}(V)$ is generated additively by the $v_{M}$, and $v_{M}$ is squarefree precisely when $M$ is a genuine subset.

A simplicial complex $K$ on $V$ consists of a finite set of faces $\sigma \subseteq V$, closed with respect to the formation of subsets $\rho \subseteq \sigma$. Alternatively, we may interpret $K$ as the set of squarefree monomials $v_{\sigma}:=\prod_{\sigma} v$, which is closed under factorisation. Every simplicial complex generates a simplicial set $K_{\bullet}$; for each $n \geq 0$, the $n$ simplices $K_{n}$ contain all $M$ of cardinality $n+1$ whose support is a face of $K$. The face and degeneracy operators delete and repeat the appropriate vertices respectively.

The Stanley-Reisner algebra $R[K]$, otherwise known as the face ring of $K$, is an important combinatorial invariant. It is defined as the quotient

$$
S_{R}(V) /\left(v_{U}: U \notin K\right),
$$

and is therefore generated additively by the simplices of $K_{\bullet}$. The algebraic properties of $R[K]$ encode a host of combinatorial features of $K$, and are discussed in detail by Bruns and Herzog [3] and Stanley [23, for example. If $K$ is the simplex on $V$, then $R[K]$ is the polynomial algebra $S_{R}(V)$.

For each $K$, Davis and Januszkiewicz defined the notion of a toric space over the cone on the barycentric subdivision of $K$, and showed that the cohomology of such a space is related to $R[K]$. The relationship follows from their application of the Borel construction, which creates a family of spaces whose cohomology ring (with coefficients in $R$ ) is isomorphic to the Stanley-Reisner algebra. All members of the family are homotopy equivalent to a certain universal example, and we refer to any space which shares their common homotopy type as a DavisJanuszkiewicz space. The isomorphisms equip $R[K]$ with a natural grading, which agrees with that induced from $S_{R}(V)$. Subsequently, Buchstaber and Panov 4] defined a CW-complex whose cohomology ring is also isomorphic to $R[K]$. They confirmed that their complex is a Davis-Januszkiewicz space by 
giving an explicit homotopy equivalence with the universal example. In [21], their space is described as the pointed colimit $\operatorname{colim}^{+} B^{K}$ of a certain CAT $(K)$ diagram $B^{K}$, which assigns the cartesian product $B^{\sigma}$ to each face $\sigma$ of $K$. Here $B$ denotes the classifying space of the circle, or $C P^{\infty}$, and $\operatorname{CAT}(K)$ is the category of faces and inclusions.

We say that a space $X$ realises the Stanley-Reisner algebra of $K$ whenever there is an algebra isomorphism $H^{*}(X ; R) \cong R[K]$. We denote the rationalisation of $X$ by $X_{0}$, and write $\operatorname{Aut}_{h o}(X)<\operatorname{End}_{h o}(X)$ for the homotopy classes of self-equivalences of $X$, considered as a subgroup of the homotopy classes of self-maps with respect to composition.

The contents of each section are as follows.

In Section 2 we describe our notation and prerequisites, including those aspects of model category theory which provide a useful context for exponential diagrams and their cohomology. We also explain why it is equally acceptable to work with the unpointed colimit $c(K):=\operatorname{colim} B^{K}$. We introduce the Stanley-Reisner algebra in Section [3, and show that the Bousfield-Kan spectral sequence for $H^{*}(c(K) ; R)$ collapses by analysing higher limits of certain $\operatorname{CAT}(K)$-diagrams. In Section 4 we apply similar techniques to prove the formality of the singular cochain algebra $C^{*}(c(K) ; R)$. Finally, we specialise to the case $R=\mathbb{Q}$ in Section [5, where we confirm that Sullivan's commutative cochain algebra $A_{P L}(c(K))$ is formal in the commutative sense. We deduce that $\mathbb{Q}[K]$ determines the rationalisation $c(K)_{0}$ whenever it is a complete intersection, and discuss the corresponding automorphism group $\operatorname{Aut}_{h o}\left(c(K)_{0}\right)$.

The authors are especially grateful to the organisers of the International Conference on Algebraic Topology, which was held on the Island of Skye in June 2001. The Conference provided the opportunity for valuable discussion with several colleagues, amongst whom Octavian Cornea, Kathryn Hess, and Taras Panov deserve special mention. Without that remarkable and stimulating environment, our work would not have begun. We are more recently indebted to John Greenlees, who brought to our attention a fundamental misconception in a previous version of this article, and to the referee, for further improvements. We also thank the London Mathematical Society, whose support of the Transpennine Topology Triangle has enabled our collaboration to continue and develop. 


\section{Background}

We begin by establishing our notation and prerequisites, recalling various aspects of Davis-Januszkiewicz spaces. We refine results of [21] in the context of model category theory, referring readers to [9] and [15] for background details. Following [25], we adopt the model category TOP of $k$-spaces and continuous functions as our topological workplace. Weak equivalences induce isomorphisms in homotopy, fibrations are Serre fibrations, and cofibrations have the left lifting property with respect to acyclic fibrations. Every function space $Y^{X}$ is endowed with the corresponding $k$-topology. Many of the spaces we consider have a distinguished basepoint $*$, and we write $\mathrm{TOP}_{+}$for the model category of pairs $(X, *)$ and basepoint preserving maps. We usually insist that the inclusion $* \rightarrow X$ be a cofibration, in which case $X$ is well-pointed; this is automatic when $X$ is a $\mathrm{CW}$-complex and $*$ its 0 -skeleton.

Given a small category $\mathrm{A}$, we refer to a covariant functor $D: \mathrm{A} \rightarrow \mathrm{R}$ as an A-diagram in R. Such diagrams are themselves the objects of a category $[A, R]$, whose morphisms are natural transformations of functors. We may interpret any object $X$ of $\mathrm{R}$ as a constant diagram, which maps every object of A to $X$ and every morphism to the identity.

Example 2.1 (1) For each integer $n \geq 0$, the category $\mathrm{ORD}(n)$ has objects $0,1, \ldots, n$, equipped with a single morphism $k \rightarrow m$ when $k \leq m$. An $\operatorname{ORD}(n)$-diagram

$$
X_{0} \stackrel{f_{1}}{\longrightarrow} X_{1} \stackrel{f_{2}}{\longrightarrow} \cdots \stackrel{f_{n}}{\longrightarrow} X_{n}
$$

consists of $n$ composable morphisms in $\mathrm{R}$.

(2) The category $\Delta$ has objects $(n):=\{0,1, \ldots, n\}$ for $n \geq 0$, and morphisms the nondecreasing functions; then $\Delta^{o p}$ - and $\Delta$-diagrams are simplicial and cosimplicial objects of $\mathrm{R}$ respectively. In particular, $\Delta: \Delta \rightarrow$ TOP is the cosimplicial space which assigns the standard $n$-simplex $\Delta(n)$ to each object $(n)$.

Given objects $X_{0}$ and $X_{1}$ of $\mathrm{R}$, we write the set of morphisms $X_{0} \rightarrow X_{1}$ as $\mathrm{R}\left(X_{0}, X_{1}\right)$; when $\mathrm{R}$ is small, the diagrams (2.2) also form a set for every $n>1$. For any $\mathrm{R}$ it is often convenient to abbreviate $\left[\Delta^{o p}, \mathrm{R}\right]$ to $\mathrm{SR}$, and write a generic simplicial object as $D_{\bullet}$. In particular, SSET denotes the category of simplicial sets $Y_{\bullet}$.

From this point on we work with an abstract simplicial complex $K$, whose faces $\sigma$ are subsets of the vertices $V$. We assume that the empty face belongs to $K$, 
and write $K^{\times}$when it is expressly omitted. The integer $|\sigma|-1$ is known as the dimension of $\sigma$, and written $\operatorname{dim} \sigma$; its maximum value $\operatorname{dim} K$ is the dimension of $K$. When $K$ contains every subset of $V$, we may call it the simplex $\Delta(V)$ on $V$. Each face of $K$ therefore determines a subsimplex $\Delta(\sigma)$, whose boundary $\partial(\sigma)$ is the complex obtained by deleting the subset $\sigma$. We also require the link $\ell_{K}(\sigma)$, whose faces consist of those $\tau \backslash \sigma$ for which $\sigma \subseteq \tau$ in $K$.

Definition 2.3 For any simplicial complex $K$, the small category $\operatorname{CAT}(K)$ has objects the faces of $K$ and morphisms the inclusions $i_{\sigma, \tau}: \sigma \subseteq \tau$. The empty face $\varnothing$ is an initial object, and the maximal faces $\mu$ admit only identity morphisms. The opposite category $\operatorname{CAT}^{o p}(K)$ has morphisms $p_{\tau, \sigma}:=i_{\sigma, \tau}^{o p}: \tau \supseteq \sigma$, and $\varnothing$ is final.

The nondegerate simplices of the nerve $N_{\bullet} \operatorname{CAT}(K)$ form the cone on the barycentric subdivision $K^{\prime}$, and those of $N_{\bullet} \mathrm{CAT}\left(K^{\times}\right)$correspond to the subcomplex $K^{\prime}$. So the classifying space $B \operatorname{CAT}(K)$, formed by realising the nerve, is a contractible $\mathrm{CW}$-complex, and $B \mathrm{CAT}\left(K^{\times}\right)$is a subcomplex homeomorphic to $K$. We shall study $\operatorname{CAT}(K)$ - and $\mathrm{CAT}^{o p}(K)$-diagrams $D$ in various algebraic and topological categories $\mathrm{R}$. Usually, $\mathrm{R}$ is pointed by an object $*$, which is both initial and final; unless stated otherwise, we then assume that $D(\varnothing)=*$.

For each face $\sigma$, the overcategories $\operatorname{CAT}(K) \downarrow \sigma$ and $\operatorname{CAT}(K) \Downarrow \sigma$ are given by restricting attention to those objects $\rho$ for which $\rho \subseteq \sigma$ and $\rho \subset \sigma$ respectively. The undercategories $\sigma \downarrow \mathrm{CAT}(K)$ and $\sigma \Downarrow \operatorname{CAT}(K)$ are defined by the objects $\sigma \subseteq \tau$ and $\sigma \subset \tau$. It follows from the definitions that

$$
\begin{aligned}
& \operatorname{CAT}(K) \downarrow \sigma=\operatorname{CAT}(\Delta(\sigma)), \quad \operatorname{CAT}(K) \Downarrow \sigma=\operatorname{CAT}(\partial(\sigma)), \\
& \sigma \downarrow \operatorname{CAT}(K)=\operatorname{CAT}\left(\ell_{K}(\sigma)\right) \text { and } \sigma \Downarrow \operatorname{CAT}(K)=\operatorname{CAT}\left(\ell_{K}(\sigma)^{\times}\right) .
\end{aligned}
$$

Dimension may be interpreted as a functor $\operatorname{dim}: \operatorname{CAT}(K) \rightarrow \operatorname{ORD}(m-1)$, which is a linear extension in the sense of [15]; thus $\mathrm{CAT}(K)$ is direct and $\mathrm{CAT}^{o p}(K)$ is inverse.

For any model category R, we may therefore follow Hovey and impose an associated model structure on the category of diagrams $[\mathrm{CAT}(K), \mathrm{R}]$. Weak equivalences $e: C \rightarrow D$ are given objectwise, in the sense that $e(\sigma): C(\sigma) \rightarrow D(\sigma)$ is a weak equivalence in R for every face $\sigma$ of $K$. Fibrations are also given objectwise. To describe the cofibrations, we consider the restrictions of $C$ and $D$ to the overcategories $\operatorname{CAT}(\partial(\sigma))$, and write $L_{\sigma} C$ and $L_{\sigma} D$ for their respective colimits; $L_{\sigma}$ is the latching functor of [15]. Then $g: C \rightarrow D$ is a cofibration whenever the induced maps

$$
C(\sigma) \amalg_{L_{\sigma} C} L_{\sigma} D \longrightarrow D(\sigma)
$$


are cofibrations in $\mathrm{R}$ for every face $\sigma$. Alternatively, the methods of Chacholski and Scherer [6] lead to the same model structure on $[\operatorname{CAT}(K), \mathrm{R}]$.

There is a dual model category structure on $\left[\mathrm{CAT}^{o p}(K), \mathrm{R}\right]$, where weak equivalences and cofibrations are given objectwise. To describe fibrations $f: C \rightarrow D$, we consider the restrictions of $C$ and $D$ to the undercategories $\operatorname{CAT}^{o p}(\partial(\sigma))$, and write $M_{\sigma} C$ and $M_{\sigma} D$ for their respective limits; $M_{\sigma}$ is the matching functor of [15. Then $f$ is a fibration whenever the induced maps

$$
C(\sigma) \longrightarrow D(\sigma) \times M_{\sigma} D M_{\sigma} C
$$

are fibrations in R for every face $\sigma$.

Definition 2.6 For any CW-pair $(X, *)$, the exponential pair of diagrams $\left(X^{K}, X_{K}\right)$ consists of functors

$$
X^{K}: \mathrm{CAT}(K) \longrightarrow \mathrm{TOP}_{+} \text {and } X_{K}: \mathrm{CAT}^{o p}(K) \longrightarrow \mathrm{TOP}+
$$

which assign the cartesian product $X^{\sigma}$ to each face $\sigma$ of $K$. The value of $X^{K}$ on $i_{\sigma, \tau}$ is the cofibration $X^{\sigma} \rightarrow X^{\tau}$, where the superfluous coordinates are set to $*$, and the value of $X_{K}$ on $p_{\tau, \sigma}$ is the fibration $X^{\tau} \rightarrow X^{\sigma}$, defined by projection. The pair are twins, in the sense that $X_{K}\left(p^{\prime}\right) \cdot X^{K}(i)=X^{K}\left(j^{\prime}\right) \cdot X_{K}(q)$ for every pullback square

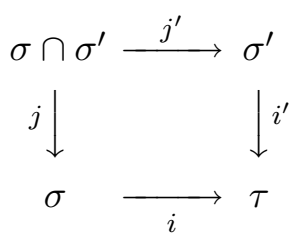

in $\operatorname{CAT}(K)$, where $p^{\prime}=\left(i^{\prime}\right)^{o p}$ and $q=j^{o p}$.

The properties of twin diagrams are analogous to those of a Mackey functor [13. They include, for example, the fact that each $X^{K}(i)$ has left inverse $X_{K}(p)$, where $p=i^{o p}$. Our applications in Theorem 3.10 are reminiscent of [16], where the acyclicity of certain Mackey functors is established.

The colimit colim $X^{K}$ is a subcomplex of $X^{V}$, whose inclusion $r$ is induced by interpreting the elements $\sigma$ of $K$ as faces of the $(m-1)$-simplex $\Delta(V)$. Composing $r$ with any of the natural maps $X^{\sigma} \rightarrow \operatorname{colim} X^{K}$ yields the standard inclusion $X^{\sigma} \rightarrow X^{V}$. We note that colim $X^{K}$ is pointed by $X^{\varnothing}$, otherwise known as the basepoint $*$, and is homeomorphic to the pointed colimit colim $^{+} X^{K}$ of 21 .

We wish to study homotopy theoretic properties of colim $X^{K}$ in favourable cases. Yet the colimit functor behaves particularly poorly in this context, because objectwise equivalent diagrams may well have homotopy inequivalent 
colimits. The standard procedure for dealing with this situation is to introduce the left derived functor, known as the homotopy colimit. Following [14, for example, hocolim $X^{K}$ may be described by the two-sided bar construction $B\left(*, \operatorname{CAT}(K), X^{K}\right)$ in TOP. We note that hocolim $X^{K}$ is also pointed, and is related to the pointed homotopy colimit hocolim ${ }^{+} X^{K}$ of [21] by the cofibre sequence

$$
\operatorname{BCAT}(K) \longrightarrow \operatorname{hocolim} X^{K} \stackrel{f}{\longrightarrow} \operatorname{hocolim}^{+} X^{K}
$$

of [2]. Since $\operatorname{BCAT}(K)$ is contractible, $f$ is a weak equivalence. We may therefore concentrate on hocolim $X^{K}$, and so avoid basepoint complications when working with function spaces in $[19]$.

Lemma 2.7 Every exponential diagram $X^{K}$ is cofibrant in $[\mathrm{CAT}(K), \mathrm{TOP}]$.

Proof The initial CAT $(K)$-diagram in TOP is the constant diagram $*$, so $X^{K}$ is cofibrant whenever the inclusion $* \rightarrow X^{K}$ is a cofibration. By (2.4), it suffices to show that the map $X^{\partial(\sigma)} \rightarrow X^{\sigma}$ is a cofibration for every face $\sigma$ of $K$. But this map includes the fat wedge in the cartesian product, and the result follows.

An immediate consequence of Lemma 2.7 is that the natural projection

$$
\operatorname{hocolim} X^{K} \longrightarrow \operatorname{colim} X^{K}
$$

is a homotopy equivalence. This exemplifies one of the fundamental properties

of the homotopy colimit functor, and is sometimes called the Projection Lemma 26.

\section{Integral cohomology and limits}

In this section we work in the category $\operatorname{MOD}_{R}$ of $R$-modules, and study the cohomology of limits of exponential diagrams $B^{K}$, where $B$ is the classifying space of the circle. For this case only, we abbreviate (2.8) to $h c(K) \rightarrow c(K)$. We focus on the relationship between the Stanley-Reisner algebra $R[K]$ and the Bousfield-Kan spectral sequence for $H^{*}(h c(K) ; R)$.

We begin by investigating the cohomology of $c(K)$. To simplify applications in later sections, we consider an arbitrary pair of twin diagrams $\left(D_{K}, D^{K}\right)$,

$$
D_{K}: \operatorname{CAT}(K) \longrightarrow \operatorname{MOD}_{R} \text { and } D^{K}: \mathrm{CAT}^{o p}(K) \longrightarrow \operatorname{MOD}_{R} .
$$


Thus $D^{K}\left(p^{\prime}\right) \cdot D_{K}(i)=D_{K}(j) \cdot D^{K}\left(q^{\prime}\right)$ for every pullback square $i \cdot j=i^{\prime} \cdot j^{\prime}$ in $\operatorname{CAT}(K)$. In particular, $D^{K}(p)$ has right inverse $D_{K}(i)$ for every morphism $p=i^{o p}$. Such pairs arise, for example, from any contravariant functor $D:$ TOP $\rightarrow$ MOD $_{R}$, by composing with the exponential twins of Definition 2.6. So $\left(D_{K}, D^{K}\right)=\left(D \cdot B_{K}, D \cdot B^{K}\right)$, and functoriality ensures the diagrams are twins. In this case we may apply $D$ to the natural maps $B^{\sigma} \rightarrow c(K) \stackrel{r}{\longrightarrow} B^{V}$, and obtain homomorphisms

$$
D\left(B^{V}\right) \stackrel{D(r)}{\longrightarrow} D(c(K)) \stackrel{h}{\longrightarrow} \lim D^{K}
$$

in $\operatorname{MOD}_{R}$.

By way of example, we consider the case $D=H^{2 j}(-, R)$, for any $j \geq 0$. For every face $\sigma$ of $K$, the space $B^{\sigma}$ is an Eilenberg-Mac Lane space $H\left(\mathbb{Z}^{\sigma}, 2\right)$, and may be expressed as the realisation of a simplicial abelian group $H_{\bullet}\left(\mathbb{Z}^{\sigma}, 2\right)$ whenever convenient [18. As a CW-complex, the cells of $B^{\sigma}$ are concentrated in even dimensions, and correspond to the simplices $v_{M}$ of $\Delta(\sigma) \bullet$. The cellular cohomology group $H^{2 j}\left(B^{\sigma} ; R\right)$ is therefore isomorphic to the free $R$-module generated by those $v_{M}$ for which $|M|=j$ and the support of $M$ is a subset of $\sigma$. The diagram $D^{K}$ of (3.1) becomes

$$
H^{2 j}\left(B^{K} ; R\right): \mathrm{CAT}^{o p}(K) \longrightarrow \operatorname{MOD}_{R},
$$

whose value on $p_{\tau, \sigma}$ is the homomorphism which fixes $v_{M}$ whenever the support of $M$ lies in $\sigma$, and annihilates it otherwise; the right inverse is the inclusion induced by $D_{K}$. When $D=H^{2 j+1}(-, R)$, the diagram is zero.

In the case of cohomology, we may combine the diagrams (3.3) into a graded version

$$
H^{*}\left(B^{K} ; R\right): \mathrm{CAT}^{o p}(K) \longrightarrow \operatorname{GMOD}_{R},
$$

taking values in the category of graded $R$-modules. The cup product on each of the constituent submodules $H^{*}\left(B^{\sigma} ; R\right)$ is given by the product of monomials $x_{L} x_{M}=x_{L+M}$, as follows from the case of a single vertex. In other words, the cohomology ring $H^{*}\left(B^{\sigma} ; R\right)$ is isomorphic to the polynomial algebra $S_{R}(\sigma)$. So $H^{*}\left(B^{K} ; R\right)$ actually takes values in the category $\mathrm{GCA}_{R}$ of graded commutative $R$-algebras, and maps the morphism $p_{\tau, \sigma}$ to the projection $S_{R}(\tau) \rightarrow S_{R}(\sigma)$; the right inverse is again inclusion.

The homomorphisms (3.2) may similarly be combined as

$$
S_{R}(V) \stackrel{r^{*}}{\longrightarrow} H^{*}(c(K) ; R) \stackrel{h}{\longrightarrow} \lim H^{*}\left(B^{K} ; R\right),
$$

where the limit is taken in $\operatorname{GMOD}_{R}$. Since (3.4) is a diagram of algebras, the limit inherits a multiplicative structure, and it is equally appropriate to interpret 
(3.5) in $\mathrm{GCA}_{R}$. The composition $h \cdot r^{*}$ is induced by the projections $S_{R}(V) \rightarrow$ $S_{R}(\sigma)$. In this case, we make one further observation.

Proposition 3.6 The homomorphism $r^{*}$ is epic, and its kernel is the ideal $\left(v_{U}: U \notin K\right)$.

Proof In each dimension $2 j$, the cells $v_{M}$ of $B^{V}$ correspond to the multisets on $V$ with $|M|=j$. The cells of $c(K)$ form a subset, given by those $M$ whose support is a face of $K$. Hence $r^{*}$ is epic, and its kernel is generated by the remaining cells. These coincide with the $2 j$-dimensional additive generators of the ideal $\left(v_{U}: U \notin K\right)$.

So there is an isomorphism $R[K] \cong H^{*}(c(K) ; R)$ of the Stanley-Reisner algebra (1.1), which plays a central rôle in [4].

Returning to our study of the twins $\left(D_{K}, D^{K}\right)$, the following definition identifies a further important property.

Definition 3.7 A diagram $F^{K}: \mathrm{CAT}^{o p}(K) \rightarrow \mathrm{MOD}_{R}$ of $R$-modules (graded or otherwise) is fat if the natural map $F^{K}(\sigma) \rightarrow \lim F^{\partial(\sigma)}$ is an epimorphism for every face $\sigma$ of $K$.

The terminology acknowledges the relationship between $\partial(\sigma)$ and the fat wedge described in Lemma 2.7.

Lemma 3.8 The twin $D^{K}$ is fat.

Proof We consider an arbitrary face $\rho$ of $K$, whose vertices we label $w_{k}$ for $0 \leq k \leq d$; thus $d=\operatorname{dim} \rho$. We write $\mu_{k}:=\rho \backslash w_{k}$ for the maximal faces of $\partial(\rho)$, and abbreviate the morphism $p_{\rho, \mu_{k}}$ to $p_{k}$ for $0 \leq k \leq d$.

The definition of $\lim$ ensures that $L:=\lim D^{\partial(\rho)}$ appears in an exact sequence

$$
0 \longrightarrow L \longrightarrow \prod_{\rho \supset \sigma} D^{K}(\sigma) \stackrel{\delta}{\longrightarrow} \prod_{\rho \supset \tau \supset \sigma} D^{K}(\sigma)
$$

where $\delta(u)(\tau \supset \sigma)=u(\sigma)-D^{K}\left(p_{\tau, \sigma}\right) u(\tau)$ for any $u \in \prod_{\rho \supset \sigma} D^{K}(\sigma)$. Hence $u \in L$ is determined by the values $u\left(\mu_{k}\right)$. The natural projection $D^{K}(\rho) \rightarrow$ $\prod_{\rho \supset \sigma} D^{K}(\sigma)$ therefore factors through $L$, and it remains for us to find $u(\rho) \in$ $D^{K}(\rho)$ such that $D^{K}\left(p_{k}\right)(u(\rho))=u\left(\mu_{k}\right)$ for every $0 \leq k \leq d$. 
We define $u(\rho):=\sum_{\rho \supset \sigma}(-1)^{|\rho \backslash \sigma|+1} D_{K}\left(i_{\sigma, \rho}\right) u(\sigma)$. The fact that $D_{K}$ and $D^{K}$ are twins implies that

$$
D^{K}\left(p_{k}\right) D_{K}\left(i_{\sigma, \rho}\right) u(\sigma)= \begin{cases}D_{K}\left(i_{\sigma \backslash w_{k}, \mu_{k}}\right) u\left(\sigma \backslash w_{k}\right) & \text { if } w_{k} \in \sigma \\ D_{K}\left(i_{\sigma, \mu_{k}}\right) u(\sigma) & \text { otherwise }\end{cases}
$$

for every $0 \leq k \leq d$; thus $D^{K}\left(p_{k}\right) u(\rho)$ is given by

$$
\sum_{\sigma \not w_{k}}(-1)^{|\rho \backslash \sigma|+1} D_{K}\left(i_{\sigma, \mu_{k}}\right) u(\sigma)+\sum_{\tau \ni w_{k}}(-1)^{|\rho \backslash \tau|+1} D_{K}\left(i_{\tau \backslash w_{k}, \mu_{k}}\right) u\left(\rho \backslash w_{k}\right) .
$$

But we may write $u(\sigma)$ as $u\left(\left(\sigma \cup w_{k}\right) \backslash w_{k}\right)$ for any $\sigma \not \ngtr w_{k}$ other than $\mu_{k}$. So the summands cancel in pairs, leaving $u\left(\mu_{k}\right)$ as required.

For cohomology, Lemma 3.8 contributes to our analysis of $c(K)$. The homotopy equivalence (2.8) provides a cohomology decomposition [8, in the sense that the cohomology algebra $H^{*}(c(K) ; R)$ may be computed by the Bousfield-Kan spectral sequence [2]

$$
E_{2}^{i, j} \Longrightarrow H^{i+j}(h c(K) ; R),
$$

where $E_{2}^{i, j}$ is isomorphic to the $i$ th derived functor $\lim ^{i} H^{j}\left(B^{K} ; R\right)$ for every $i, j \geq 0$. The vertical edge homomorphism coincides with the map $h$ of (3.5). Lemma 3.8 is required for our computation of these limits, and Corollary 3.12 will confirm that the cohomology decomposition is sharp in Dwyer's language. Our proof uses the calculus of functors and their limits; the appropriate prerequisites may be deduced from Gabriel and Zisman [12, Appendix II §3], by dualising their results for colimits.

In particular, we follow 12 (as expounded in 20, for example) by calculating $\lim ^{i} D^{K}$ as the $i$ th cohomology group of a certain cochain complex $\left(C^{*}\left(D^{K}\right), \delta\right)$ of $R$-modules. The groups are defined by

$$
C^{n}\left(D^{K}\right):=\prod_{\sigma_{0} \supseteq \ldots \supseteq \sigma_{n}} D^{K}\left(\sigma_{n}\right) \quad \text { for } n \geq 0,
$$

and the differential $\delta:=\sum_{k=0}^{n}(-1)^{k} \delta^{k}$ is defined on $u \in C^{n}\left(D^{K}\right)$ by

$$
\delta^{k}(u)\left(\sigma_{0} \supseteq \cdots \supseteq \sigma_{n+1}\right):= \begin{cases}u\left(\sigma_{0} \supseteq \cdots \supseteq \widehat{\sigma}_{k} \supseteq \cdots \supseteq \sigma_{n+1}\right) & \text { for } k \neq n+1 \\ D^{K}\left(p_{\sigma_{n}, \sigma_{n+1}}\right) u\left(\sigma_{0} \supseteq \cdots \supseteq \sigma_{n}\right) & \text { for } k=n+1 .\end{cases}
$$

We may replace $C^{*}\left(D^{K}\right)$ by its quotient $N^{*}\left(D^{K}\right)$ of normalised cochains, for which the faces $\sigma_{0} \supset \cdots \supset \sigma_{n}$ are required to be distinct. 
Lemma 3.9 Given a maximal face $\mu$ of $K$, and a diagram $D: \mathrm{CAT}^{o p}(K) \rightarrow$ $\operatorname{MOD}_{R}$ such that $D(\sigma)=0$ for all $\sigma \neq \mu$, then

$$
\lim ^{i} D= \begin{cases}D(\mu) & \text { for } i=0 \\ 0 & \text { for } i>0\end{cases}
$$

Proof Since $\mu$ is maximal, the only morphism $\sigma \supseteq \mu$ is the identity. So the normalised chain complex $N^{*}(D)$ is $D(\mu)$ in dimension 0 , and 0 in higher dimensions, as required.

Theorem 3.10 For any fat diagram $F^{K}: \operatorname{CAT}^{o p}(K) \rightarrow \operatorname{MOD}_{R}$, we have that $\lim ^{i} F^{K}=0$ for all $i>0$; in particular, $\lim ^{i} D^{K}=0$ for every twin $D^{K}$.

Proof We proceed by induction on the total number of faces $f(K)$; the result obviously holds for the initial example $K=\varnothing$, where $f(K)=0$. Our inductive hypothesis is that $\lim ^{i} F^{K}$ vanishes whenever $K$ satisfies $f(K) \leq f$.

We therefore consider an arbitrary complex $K$ with $f(K)=f+1$, and write $J \subset K$ for the subcomplex obtained by deleting a single maximal face $\mu$. The inclusion of $J$ defines a functor $G: \mathrm{CAT}^{o p}(J) \rightarrow \mathrm{CAT}^{o p}(K)$, whose induced functor $G^{*}:\left[\mathrm{CAT}^{o p}(K), \mathrm{MOD}_{R}\right] \rightarrow\left[\mathrm{CAT}^{o p}(J), \mathrm{MOD}_{R}\right]$ acts by restriction, and admits a right adjoint $G_{*}$, known as the right Kan extension [17. In particular, $G_{*} F^{J}$ is given on $\sigma \in K$ by $\lim F^{\partial(\mu)}$ when $\sigma=\mu$, and $F^{\sigma}$ otherwise.

But $F^{\mu} \rightarrow \lim F^{\partial(\mu)}$ is an epimorphism, by Lemma 3.8. so the natural transformation $F^{K} \rightarrow G_{*} F^{J}$ is epic on every face of $K$, and its kernel $H$ is zero on every face except $\mu$. We acquire a short exact sequence of functors

$$
0 \longrightarrow H \longrightarrow F^{K} \longrightarrow G_{*} F^{J} \longrightarrow 0
$$

which induces a long exact sequence of higher limits. By Lemma 3.9, this collapses to a sequence of isomorphisms

$$
\lim ^{i} F^{K} \cong \lim ^{i} G_{*} F^{J}
$$

for $i \geq 1$. We now apply the composition of functors spectral sequence [5], 12]

$$
\lim ^{n} G_{*}^{i} F^{J} \Longrightarrow \lim ^{n+i} F^{J} .
$$

Here $G_{*}^{i}$ denotes the $i$ th derived functor of $G_{*}$; it may be evaluated on any face $\sigma$ of $K$ as $\lim ^{i} F^{\partial(\sigma)}$, and therefore vanishes for $i>0$, by inductive hypothesis. So the spectral sequence collapses onto the first row of the $E^{2}$ page, from which we obtain isomorphisms $\lim ^{n} G_{*} F^{J} \cong \lim ^{n} F^{J}$ for all $n \geq 0$. Since the inductive hypothesis applies to $J$, we deduce that $\lim ^{n} G_{*} F^{J}=0$ for every $n>0$. Combining this with (3.11) concludes the proof. 
Corollary 3.12 The Bousfield-Kan spectral sequence for $B^{K}$ collapses at the $E_{2}$ page; it is concentrated along the vertical axis, and given by

$$
\lim ^{i} H^{j}\left(B^{K} ; R\right)= \begin{cases}\lim H^{j}\left(B^{K} ; R\right) & \text { if } i=0 \\ 0 & \text { otherwise. }\end{cases}
$$

Proof The result follows immediately from Theorem 3.10 by letting $D^{K}$ be $H^{j}(-; R)$ for every $j \geq 0$.

Corollary 3.12 confirms that the edge homomorphism $h$ is an isomorphism in $\mathrm{GCA}_{R}$. When combined with (3.5) and Proposition 3.6] it implies that the natural map

$$
R[K] \cong H^{*}(c(K) ; R) \stackrel{h}{\longrightarrow} \lim H^{*}\left(B^{K} ; R\right),
$$

which is induced by the projections $R[K] \rightarrow S_{R}(\sigma)$, is also an isomorphism. This may be proven directly, by refining the methods of Proposition 3.6.

\section{Integral formality}

In this section we study the formality of $c(K)$ over our arbitrary commutative ring $R$, and construct a zig-zag of weak equivalences between the singular cochain algebra $C^{*}(c(K) ; R)$ and its cohomology ring.

We work in the model category $\mathrm{DGA}_{R}$ of differential graded $R$-algebras, whose differentials have degree +1 ; morphisms which induces isomorphisms in cohomology are known as quasi-isomorphisms. The model structure arises by interpreting $\mathrm{DGA}_{R}$ as the category of monoids in the monoidal model category DGMOD $_{R}$ of unbounded cochain complexes over $R$. The latter is isomorphic to Hovey's category of unbounded chain complexes [15], and the model structure is induced on $\mathrm{DGA}_{R}$ by checking that it satisfies the monoid axiom of 22. As Schwede and Shipley confirm, weak equivalences are quasi-isomorphisms and fibrations are epimorphisms. Cofibrations are defined by the appropriate lifting property, and are necessarily degreewise split injections. We emphasise that the objects of $\mathrm{DGA}_{R}$ need not be commutative.

A differential graded $R$-algebra $C^{*}$ is formal in $\mathrm{DGA}_{R}$ whenever there is a zig-zag of quasi-isomorphisms

$$
H\left(C^{*}\right) \stackrel{\sim}{\longrightarrow} \cdots \stackrel{\sim}{\longleftarrow} C^{*}
$$

in $\mathrm{DGA}_{R}$, where we follow the standard convention of assigning the zero differ-

ential to the cohomology algebra $H\left(C^{*}\right)$. Our aim is to show that the cochain 
algebra $C^{*}(c(K) ; R)$ is always formal in DGA $R$. This extends Franz's result [11], which only applies to complexes arising from smooth fans.

We begin by choosing $D$ to be the $j$-dimensional cochain functor $C^{j}(-; R)$ in (3.1), thus creating twin diagrams $\left(C^{j}\left(B_{K} ; R\right), C^{j}\left(B^{K} ; R\right)\right)$ for each $j \geq 0$. As in (3.4), we may consider the graded version $C^{*}(-; R)$ in $\operatorname{DGMOD}_{R}$. In fact its values are always $R$-algebras, with respect to the cup product of cochains. The product is not commutative, but the procedure for forming the limit of a DGA $_{R}$-diagram remains the same; work in $\operatorname{DGMOD}_{R}$, and superimpose the induced multiplicative structure.

For the Eilenberg-Mac Lane space $B$, we let $v$ denote a generator of $H^{2}(B ; R)$, which is isomorphic to $R$. We choose a cocycle $\psi_{v}$ representing $v$ in $C^{2}(B ; R)$, and define a homomorphism $\psi: H^{*}(B ; R) \rightarrow C^{*}(B ; R)$ by $\psi\left(v^{k}\right)=\left(\psi_{v}\right)^{k}$, for all $k \geq 0$. By construction, $\psi$ is multiplicative, and is a quasi-isomorphism in $\operatorname{DGA}_{R}$. In order to extend this procedure we introduce a quasi-isomorphism $\kappa$, defined by composition with the Künneth isomorphism as

$$
H^{*}\left(B^{V} ; R\right) \stackrel{\cong}{\longrightarrow} H^{*}(B ; R)^{\otimes V} \stackrel{\psi^{\otimes}}{\longrightarrow} C^{*}(B ; R)^{\otimes V} ;
$$

$\kappa$ is also multiplicative. There is a further zig-zag of quasi-isomorphims

$$
\operatorname{Hom}\left(C_{*}(B) ; R\right)^{\otimes V} \longrightarrow \operatorname{Hom}\left(C_{*}(B)^{\otimes V} ; R\right) \stackrel{e z}{\longleftarrow} \operatorname{Hom}\left(C_{*}\left(B^{V}\right) ; R\right),
$$

in which $e z$ is the dual of Eilenberg-Zilber map. Both arrows lie in $\mathrm{DGA}_{R}$, so we may combine (4.2) and (4.3) to create the zig-zag

$$
H^{*}\left(B^{V} ; R\right) \stackrel{\kappa}{\longrightarrow} C^{*}(B ; R)^{\otimes V} \longrightarrow \operatorname{Hom}\left(C_{*}(B)^{\otimes V} ; R\right) \stackrel{e z}{\longleftarrow} C^{*}\left(B^{V} ; R\right) .
$$

This confirms that $C^{*}\left(B^{n} ; R\right)$ is formal in $\mathrm{DGA}_{R}$ for every $n \geq 1$.

For each $B^{\sigma}$, we may project (4.4) onto the corresponding zig-zag of quasiisomorphisms. The results are compatible by naturality, and so provide morphisms

$$
H^{*}\left(B^{K} ; R\right) \stackrel{\kappa}{\longrightarrow} C^{*}(B ; R)^{\otimes K} \longrightarrow \operatorname{Hom}\left(C_{*}(B)^{\otimes K} ; R\right) \stackrel{e z}{\longleftarrow} C^{*}\left(B^{K} ; R\right)
$$

of $\mathrm{CAT}^{o p}(K)$-diagrams. Taking limits in $\mathrm{DGA}_{R}$ yields the zig-zag

$$
\begin{aligned}
& \lim H^{*}\left(B^{K} ; R\right) \stackrel{\kappa}{\longrightarrow} \lim C^{*}(B ; R)^{\otimes K} \longrightarrow \\
& \lim \left(\operatorname{Hom}\left(C_{*}(B)^{\otimes K} ; R\right)\right) \stackrel{e z}{\longleftarrow} \lim C^{*}\left(B^{K} ; R\right) .
\end{aligned}
$$

Lemma 4.6 All three homomorphisms of 4.5) are quasi-isomorphisms in $\mathrm{DGA}_{R}$. 
Proof A diagram $D^{K}: \mathrm{CAT}^{o p}(K) \rightarrow \mathrm{DGA}_{R}$ is fibrant whenever the projection onto the constant diagram 0 is a fibration. By (2.5) this occurs precisely when $D^{K}$ is fat, and therefore holds for $H^{*}\left(B^{K} ; R\right)$ and $C^{*}\left(B^{K} ; R\right)$ by Lemma 3.8 it follows for $H^{*}(B ; R)^{\otimes K}$ by the Künneth isomorphism. So far as $C^{*}(B ; R)^{\otimes K}$ is concerned, we note that singular cochains determine a pair of twin diagrams $\left(C_{\otimes K}, C^{\otimes K}\right)$ in $\mathrm{DGA}_{R}$. Both functors assign $C^{*}(B ; R)^{\otimes \sigma}$ to the face $\sigma$. The value of $C_{\otimes K}$ on $i_{\sigma, \tau}$ is the inclusion $C^{*}(B ; R)^{\otimes \sigma} \rightarrow C^{*}(B ; R)^{\otimes \tau}$, and the value of $C^{\otimes K}$ on $p_{\tau, \sigma}$ is the projection $C^{*}(B ; R)^{\otimes \tau} \rightarrow C^{*}(B ; R)^{\otimes \sigma}$; the latter requires the augmentation induced by the base point of $B$. Hence $C^{\otimes K}$ is also fat, and $C^{*}(B ; R)^{\otimes K}$ is fibrant. Similar remarks apply to $\operatorname{Hom}\left(C_{*}(B)^{\otimes K} ; R\right)$.

The homomorphisms in question are therefore objectwise equivalences of fibrant diagrams, and induces weak equivalences of limits by [15].

Lemma 4.7 The natural homomorphism $g: C^{*}(c(K) ; R) \rightarrow \lim C^{*}\left(B^{K} ; R\right)$ is a quasi-isomorphism in $\mathrm{DGA}_{R}$.

Proof The edge isomorphism $h$ of (3.5) and Corollary 3.12 factorises as

$$
H\left(C^{*}(c(K) ; R)\right) \stackrel{H(g)}{\longrightarrow} H\left(\lim C^{*}\left(B^{K} ; R\right)\right) \stackrel{l}{\longrightarrow} \lim H^{*}\left(B^{K} ; R\right)
$$

in $\mathrm{DGA}_{R}$, where $l$ is induced by the collection of compatible homomorphisms

$$
H\left(\lim C^{*}\left(B^{K} ; R\right)\right) \longrightarrow H^{*}\left(B^{\sigma} ; R\right) .
$$

Now let $d$ be the differential on $C^{j}(-; R)$ for every $j \geq 0$, and define the cycle and boundary functors $Z^{j}, I^{j}: \mathrm{TOP} \rightarrow \mathrm{MOD}_{R}$ as the kernel and image of $d$ respectively. They determine twin diagrams, and therefore fat functors $Z^{K}, I^{K}: \operatorname{CAT}^{o p}(K) \rightarrow \operatorname{MOD}_{R}$. Theorem 3.10 confirms that $\lim ^{i} Z^{j}\left(B^{K} ; R\right)=$ $\lim ^{i} I^{j}\left(B^{K} ; R\right)=0$ for all $i>0$ and $j \geq 0$. It follows immediately that $l$ is an isomorphism, and therefore that $H(g)$ is an isomorphism, as sought.

We may now complete our analysis of $C^{*}(c(K) ; R)$.

Theorem 4.8 The differential graded $R$-algebra $C^{*}(c(K) ; R)$ is formal in $\mathrm{DGA}_{R}$.

Proof Combining Corollary 3.12 with Lemmas 4.6 and 4.7 yields a zig-zag

$$
H^{*}(c(K) ; R) \stackrel{h}{\longrightarrow} \lim H^{*}\left(B^{K} ; R\right) \longrightarrow \ldots \longleftarrow \lim C^{*}\left(B^{K} ; R\right) \stackrel{g}{\longleftarrow} C^{*}(c(K) ; R)
$$

of quasi-isomorphisms, as required by (4.1). 
Remark 4.9 The proof of Theorem 4.8 extends to exponential diagrams $X^{K}$ for which $C^{*}(X ; R)$ is formal in $\mathrm{DGA}_{R}$ and the Künneth isomorphism $H^{*}\left(X^{V} ; R\right) \cong H^{*}(X ; R)^{\otimes V}$ holds. We replace $\psi$ in (4.4) by the corresponding zig-zag $H^{*}(X ; R) \stackrel{\sim}{\longrightarrow} \cdots \stackrel{\sim}{\longleftarrow} C^{*}(X ; R)$ of quasi-isomorphisms, and repeat the remainder of the argument above.

\section{Rational formality}

In our final section we turn to the rational case $R=\mathbb{Q}$, and confirm the formality of Sullivan's algebra of rational cochains on $c(K)$ in the commutative setting. This involves stricter conditions than those for general $R$, and has deeper topological consequences. In particular, it leads us to a minimal model whenever $\mathbb{Q}[K]$ is a complete intersection ring, and thence to rational uniqueness. We refer readers to Bousfield and Gugenheim [1] for details of the model category of differential graded commutative $\mathbb{Q}$-algebras, and to Félix, Halperin and Thomas [10] for background information on rational homotopy theory.

We begin by replacing $C^{*}(X ; R)$ with Sullivan's rational algebra $A_{P L}(X)$ of polynomial forms [10]. The commutativity of the latter is crucial, and suggests we work in the category DGCA $\mathbb{Q}$ of differential graded commutative $\mathbb{Q}$-algebras 1]. The existence of a model structure is assured by working over a field; as before, weak equivalences are quasi-isomorphisms, fibrations are epimorphisms, and cofibrations are defined by the appropriate lifting properties.

For each $s \geq 0$, we write the differential algebra of rational polynomial forms on the standard $s$-simplex as $\nabla_{s}(*)$. It is an object of $\mathrm{DGCA}_{\mathbb{Q}}$. For each $t \geq 0$, the forms of dimension $t$ define a simplicial vector space $\nabla_{\bullet}(t)$ over $\mathbb{Q}$, and $\nabla \bullet(*)$ becomes a simplicial object in $\mathrm{DGCA}_{\mathbb{Q}}$. So

$$
A^{*}\left(Y_{\bullet}\right):=\operatorname{SSET}\left(Y_{\bullet}, \nabla_{\bullet}(*)\right)
$$

is also an object of $\mathrm{DGCA}_{\mathbb{Q}}$, which is weakly equivalent to the normalised cochain complex $N^{*}\left(Y_{\bullet} ; \mathbb{Q}\right)$. Then $A_{P L}(X)$ is defined as $A^{*}\left(S_{\bullet} X\right)$, where $S_{\bullet}$ denotes the total singular complex functor тоP $\rightarrow$ SSET. The PL de Rham Theorem 1] asserts that the cohomology algebra $H\left(A_{P L}(X)\right)$ is naturally isomorphic to $H^{*}(X ; \mathbb{Q})$. As usual, we invest cohomology algebras with the zero differential.

A differential graded commutative $\mathbb{Q}$-algebras $A^{*}$ is formal in $\mathrm{DGCA}_{\mathbb{Q}}$ whenever there is a zig-zag of quasi-isomorphisms

$$
H\left(A^{*}\right) \stackrel{\sim}{\longrightarrow} \cdots \stackrel{\sim}{\longleftarrow} A^{*}
$$


in $\mathrm{DGCA}_{\mathbb{Q}}$. A topological space $X$ is rationally formal whenever $A_{P L}(X)$ satisfies (5.1).

For any such $X$, a minimal Sullivan model may be constructed directly from the algebra $H^{*}(X ; \mathbb{Q})$. Our remaining goal is therefore to show that $c(K)$ is rationally formal, and to consider the implications for the uniqueness of spaces $X$ realising $\mathbb{Q}[K]$. The proof parallels that for general $R$, but the need to respect commutativity forces several changes of detail.

We choose $D$ to be $A_{P L}$ in (3.1), creating twin diagrams $\left(A_{P L}\left(B_{K}\right), A_{P L}\left(B^{K}\right)\right)$ in $\mathrm{DGCA}_{\mathbb{Q}}$. As before, we form limits by working in $\mathrm{DGMOD}_{Q}$, and superimposing the induced multiplicative structure. Applying cohomology yields the twins $\left(H^{*}\left(B_{K} ; \mathbb{Q}\right), H^{*}\left(B^{K} ; \mathbb{Q}\right)\right)$, whose value on each face $\sigma$ is $S_{\mathbb{Q}}(\sigma)$ in $\operatorname{DGMOD}_{Q}$. Both $A_{P L}\left(B^{K}\right)$ and $H^{*}\left(B^{K} ; \mathbb{Q}\right)$ are fat, by Lemma 3.8 .

Using the fact that $H\left(A_{P L}\left(B^{V}\right)\right)$ is isomorphic to $S_{\mathbb{Q}}(V)$, we choose cocycles $\phi_{j}$ in $A_{P L}\left(B^{V}\right)$ representing $v_{j}$ for every $1 \leq j \leq m$. We may then define a homomorphism

$$
\phi: H^{*}\left(B^{V} ; \mathbb{Q}\right) \longrightarrow A_{P L}\left(B^{V}\right)
$$

by $\phi\left(v_{j}\right)=\phi_{j}$, because $A_{P L}\left(B^{V}\right)$ is commutative. Moreover, $\phi$ is a quasiisomorphism, reflecting the rational formality of the Eilenberg-Mac Lane space $H\left(\mathbb{Z}^{V} ; 2\right)$. By restriction, we interpret the $\phi_{j}$ as cocycles in $A_{P L}\left(B^{\sigma}\right)$ for every face $\sigma$. They then represent $v_{j}$ in $S_{\mathbb{Q}}(\sigma)$ when $\sigma$ contains $v_{j}$, and 0 otherwise. We obtain compatible quasi-isomorphisms on each $B^{\sigma}$, which combine to create a map

$$
\phi: H^{*}\left(B^{K} ; \mathbb{Q}\right) \longrightarrow A_{P L}\left(B^{K}\right)
$$

of $\operatorname{CAT}^{o p}(K)$-diagrams in $\mathrm{DGCA}_{\mathbb{Q}}$. It is an objectwise weak equivalence. Taking limits yields a homomorphism

$$
l(\phi): \lim H^{*}\left(B^{K} ; \mathbb{Q}\right) \longrightarrow \lim A_{P L}\left(B^{K}\right)
$$

of differential graded commutative algebras over $\mathbb{Q}$.

Lemma 5.3 The homomorphism $l(\phi)$ is a quasi-isomorphism in DGCA $\mathbb{Q}$.

Proof Both diagrams are fat, and therefore fibrant by (2.5). So $\phi$ induces a weak equivalence of limits.

Because the $B^{\sigma}$ are Eilenberg-Mac Lane spaces, it is convenient to complete our proof of Theorem 5.5] in terms of simplicial sets. We may then take advantage of the fact that $A^{*}$ converts colimits in SSET to limits in $\mathrm{DGCA}_{\mathbb{Q}}$, for reasons which are purely set-theoretic. 
We denote the realisation functor $\operatorname{SSET} \rightarrow$ TOP by $|-|$. Given an arbitrary simplicial set $Y_{\bullet}$, there is a quasi-isomorphism

$$
A_{P L}\left(\left|Y_{\bullet}\right|\right)=A^{*}\left(S_{\bullet}\left|Y_{\bullet}\right|\right) \stackrel{\sim}{\longrightarrow} A^{*}\left(Y_{\bullet}\right),
$$

induced by the natural equivalence $Y_{\bullet} \rightarrow S_{\bullet}\left|Y_{\bullet}\right|$. For each face $\sigma$ of $K$, we choose $\left|H_{\bullet}\left(\mathbb{Z}^{\sigma} ; 2\right)\right|$ as our model for $B^{\sigma}$; it is well-pointed, by the cofibration induced by the inclusion of the trivial subgroup $\{0\} \rightarrow \mathbb{Z}^{\sigma}$. We write $H_{\bullet}^{K}$ for the corresponding diagram of simplicial sets, which takes the value $H_{\bullet}\left(\mathbb{Z}^{\sigma} ; 2\right)$ on $\sigma$.

Theorem 5.5 The space $c(K)$ is rationally formal.

Proof Since realisation is left adjoint to $S_{\bullet}$, it commutes with colimits. So we may write

$$
c(K)=\operatorname{colim}\left|H_{\bullet}^{K}\right| \cong\left|\operatorname{colim} H_{\bullet}^{K}\right|,
$$

where the second colimit is taken in SSET. Applying (5.4) gives a zig-zag

$$
\lim A_{P L}\left(B^{K}\right) \stackrel{\sim}{\longrightarrow} \lim A^{*}\left(H_{\bullet}^{K}\right) \stackrel{\cong}{\longrightarrow} A^{*}\left(\operatorname{colim} H_{\bullet}^{K}\right) \stackrel{\sim}{\longleftarrow} A_{P L}(c(K)),
$$

where the central isomorphism follows from the property of $A^{*}$ described above. Combining (5.6) with Corollary 3.12 and Lemma 5.3 yields a zig-zag

$$
H^{*}(c(K) ; \mathbb{Q}) \stackrel{h}{\longrightarrow} \lim H^{*}\left(B^{K} ; \mathbb{Q}\right) \stackrel{l(\phi)}{\longrightarrow} \lim A_{P L}\left(B^{K}\right) \stackrel{\sim}{\longrightarrow} \cdots \stackrel{\sim}{\longleftarrow} A_{P L}(c(K))
$$

of quasi-isomorphisms in DGCA $\mathbb{Q}$. The result follows from (5.1).

Remark 5.7 By analogy with Remark 4.9, the proof of Theorem [5.5] extends to exponential diagrams $X^{K}$ for which $X$ is rationally formal; the Künneth isomorphism holds automatically, because we are working over $\mathbb{Q}$. The product $A_{P L}(X)^{\otimes V} \rightarrow A_{P L}\left(X^{V}\right)$ of the maps induced by projection is a quasiisomorphism, and is natural with respect to projection and inclusion of coordinates. So we may replace $\phi$ in (5.2) by the corresponding zig-zag of quasiisomorphisms

$$
H^{*}\left(X^{V} ; \mathbb{Q}\right) \stackrel{\cong}{\longrightarrow} H^{*}(X ; \mathbb{Q})^{\otimes V} \stackrel{\sim}{\longrightarrow} \cdots \stackrel{\sim}{\longleftarrow} A_{P L}(X)^{\otimes V} \stackrel{\sim}{\longrightarrow} A_{P L}\left(X^{V}\right)
$$

and proceed with the remainder of the argument above.

Theorem [5.5 confirms that a minimal Sullivan model for $c(K)$ may be constructed directly from $\mathbb{Q}[K]$. It consists of an acyclic fibration

$$
\eta:\left(S_{\mathbb{Q}}(W(K)), d\right) \longrightarrow H^{*}(c(K) ; \mathbb{Q}),
$$


where $W(K)$ is an appropriately graded set of generators (necessarily exterior in odd dimensions), and provides a cofibrant replacement for $H^{*}(c(K) ; \mathbb{Q})$ in $\mathrm{DGCA}_{\mathbb{Q}}$. In general, $W(K)$ is not easy to describe, although special cases such as Example 5.10 below are straightforward, and lead to our uniqueness result. The properties of $W(K)$ are linked to those of the loop space $\Omega c(K)$, whose study was begun in 21]; we expect to return to this relationship in future.

The principal calculational tool of rational homotopy theory is the Sullivan-de Rham equivalence of homotopy categories, which asserts that Bousfield and Gugenheim's adjoint pair of derived functors

$$
h o \operatorname{SSET}_{\mathbb{Q}} \rightleftarrows h o \operatorname{DGCA}_{\mathbb{Q}}
$$

restrict to inverse equivalences between certain full subcategories [1. These are given by nilpotent simplicial sets of finite type, and algebras which are equivalent to minimal algebras with finitely many generators in each dimension.

In particular, the equivalence identifies homotopy classes of maps $\left[c(K)_{0}, c(L)_{0}\right]$ with homotopy classes of morphisms $\left[S_{\mathbb{Q}}(W(L)), S_{\mathbb{Q}}(W(K))\right]$. Since every object of DGCA $\mathbb{Q}$ is fibrant, it suffices to consider homotopy classes of the form $\left[S_{\mathbb{Q}}(W(L)), H^{*}(c(K) ; \mathbb{Q})\right] ;$ of course $S_{\mathbb{Q}}(W(L))$ cannot be substituted similarly, because $H^{*}(c(L) ; \mathbb{Q})$ is not usually cofibrant. Nevertheless, the function

$$
\left[S_{\mathbb{Q}}(W(L)), H^{*}(c(K) ; \mathbb{Q})\right] \longrightarrow \operatorname{GCA}_{\mathbb{Q}}\left(H^{*}(c(L) ; \mathbb{Q}), H^{*}(c(K) ; \mathbb{Q})\right)
$$

induced by taking cohomology is always surjective, and it would be of interest to understand its kernel.

Example 5.10 Let $(\lambda(k): 1 \leq k \leq t)$ be a sequence of disjoint subsets of $V$, where $\lambda(k)$ has cardinality $n(k)$, and define $L$ to be the subcomplex of $\Delta(V)$ obtained by deleting all faces containing one or more of the $\lambda(k)$. We write $\lambda:=\cup_{k} \lambda(k)$, and $|\lambda|=n$. Over any commutative ring $R$, the Stanley-Reisner algebra $R[L]$ is given by quotienting out the regular sequence $v_{\lambda(1)}, \ldots v_{\lambda(t)}$ from $S_{R}(V)$.

Over $\mathbb{Q}$, the generating set $W(L)$ of (5.8) consists of $V$ in dimension 2 , and elements $w(k)$ in dimension $2 n(k)-1$, for $1 \leq k \leq t$; the differential is given by $d v_{j}=0$ for all $j$, and $d w(k)=v_{\lambda(k)}$. The fibration $\eta$ identifies the vertices $V$ in dimension 2 , and annihilates every $w(k)$.

Since the elements $w(k)$ are odd dimensional, every DGCA $_{\mathbb{Q}}$-morphism

$$
S_{\mathbb{Q}}(W(L)) \rightarrow H^{*}(c(K) ; \mathbb{Q})
$$


is determined by its effect on $V$, and the function (5.9) is bijective. It follows that $\operatorname{Aut}_{h o}\left(c(L)_{0}\right)$ (as defined in Section (1I) is isomorphic to the group of algebra automorphisms $\operatorname{Aut}(\mathbb{Q}[L])$, and is therefore a subgroup of $G L(m, \mathbb{Q})$. It contains all matrices of the form $\left(\begin{array}{cc}M & 0 \\ N & \Sigma\end{array}\right)$, where $M \in G L(m-n, \mathbb{Q})$ acts on $\mathbb{Q}^{V \backslash \lambda}$, and $\Sigma$ permutes the elements of $\lambda$. The permutations act on the elements of each individual $\lambda(k)$, and interchange those $\lambda(k)$ which are of common cardinality. We conjecture that every automorphism of $\mathbb{Q}[L]$ may be represented by such a matrix.

It is convenient to refer to $L$ as a complete intersection complex whenever $\mathbb{Q}[L]$ is a complete intersection ring. A straightforward application of 33. Theorem 2.3.3] shows that this occurs if and only if $L$ takes the form of Example 5.10.

Our final result concerns uniqueness, and is a consequence of the fact that complete interseection complexes are a special case of Sullivan's examples 24, page 317]. Following Sullivan, it may be summarised by the statement that $c(L)$ and $\mathbb{Q}[L]$ are intrinsically formal for any such $L$.

Proposition 5.11 Let $X$ be a nilpotent $C W$-complex of finite type, which realises a complete intersection ring $\mathbb{Q}[L]$; then the rationalisations of $X$ and $c(L)$ are homotopy equivalent.

Proof We write the generators of the cohomology algebra $H^{*}(X ; \mathbb{Q})$ as $v_{j}^{\prime}$, where $1 \leq j \leq m$, and choose representing cocycles $\phi_{j}$ in $A_{P L}(X)$. Each monomial $v_{M}^{\prime}$ is therefore represented by the corresponding product $\phi_{M}$. It follows that $\phi_{\lambda(k)}$ is a coboundary, and there exist elements $\theta(k)$ such that $d \theta(k)=\phi_{\lambda(k)}$ in $A_{P L}(X)$ for all $1 \leq k \leq t$.

We define a DGCA $\mathbb{Q}$-morphism $\eta: S_{\mathbb{Q}}(W(L)) \rightarrow A_{P L}(X)$ by setting $\eta\left(v_{j}\right):=\phi_{j}$ and $\eta(w(k)):=\theta(k)$, for $1 \leq j \leq m$ and $1 \leq k \leq t$ resepectively. So $\eta$ is a weak equivalence, and is a minimal model for $X$. Hence $X$ and $c(L)$ have isomorphic minimal models, and the result follows. 


\section{References}

[1] A K Bousfield, Victor K A M Gugenheim, On PL De Rham theory and rational homotopy type, Mem. Amer. Math. Soc. 8 (1976) no. 179 MathReview

[2] A K Bousfield, Daniel M Kan, Homotopy Limits, Completions and Localizations, Lecture Notes in Mathematics 304, Springer-Verlag (1972) MathReview

[3] Winfried Bruns, Hans Jørgen Herzog, Cohen-Macaulay rings, second edition, Cambridge Studies in Advanced Mathematics 39, Cambridge University Press (1998) MathReview

[4] Victor M Buchstaber, Taras E Panov, Torus Actions and Their Applications in Topology and Combinatorics, University Lecture Series 24, Amer. Math. Soc. (2002) MathReview

[5] Henri Cartan, Samuel Eilenberg, Homological Algebra, Princeton University Press (1956) MathReview

[6] Wojciech Chachólski, Jérôme Scherer, Homotopy theory of diagrams, Mem. Amer. Math. Soc. 155, (2002) no. 736 MathReview

[7] Michael W Davis, Tadeusz Januszkiewicz, Convex Polytopes, Coxeter Orbifolds and Torus Actions, Duke Math. J. 62 (1991) 417-451 MathReview

[8] William G Dwyer, Classifying Spaces and Homology Decompositions, from: "Homotopy Theoretic Methods in Group Cohomology", Advanced Courses in Mathematics CRM Barcelona, Birkhäuser (2001) 1-53

[9] William G Dwyer, J Spaliński, Homotopy Theories and Model Categories, from: "Handbook of Algebraic Topology", (Ioan M James, editor), Elsevier (1995) 73-126 MathReview

[10] Yves Félix, Stephen Halperin, Jean-Claude Thomas, Rational Homotopy Theory, Graduate Texts in Mathematics 205, Springer-Verlag (2001) MathReview

[11] Matthias Franz, On the integral cohomology of smooth toric varieties (2003), arXiv:math.AT/0308253

[12] Peter Gabriel, Michel Zisman, Calculus of Fractions and Homotopy Theory, Ergebnisse series 35, Springer-Verlag (1967) MathReview

[13] James A Green, Axiomatic representation theory for finite groups, J. Pure Appl. Algebra 1 (1971) 41-77 MathReview

[14] Jens Hollender, Rainer M Vogt, Modules of topological spaces, applications to homotopy limits and $E_{\infty}$ structures, Arch. Math. 59 (1992) 115-129 MathReview

[15] Mark Hovey, Model Categories, Mathematical Surveys and Monographs 63, Amer. Math. Soc. (1999) MathReview 
Homotopy types, formality and rationalisation

[16] Stefan Jackowski, James McClure, Homotopy decompositions of classifying spaces via elementary abelian subgroups, Topology 31 (1992) 113-132 MathReview

[17] Saunders MacLane, Categories for the Working Mathematician, Graduate Texts in Mathematics 5, Springer-Verlag (1971) MathReview

[18] J Peter May, Simplicial Objects in Algebraic Topology, Van Nostrand Mathematical Studies 11, Van Nostrand Reinhold (1967) MathReview

[19] Dietrich Notbohm, Nigel Ray, On Davis-Januszkiewicz Homotopy Types II; Completion and Globalisation, in preparation

[20] Bob Oliver, Higher limits via Steinberg representations, Comm. Algebra 22 (1994) 1381-1393 MathReview

[21] Taras Panov, Nigel Ray, Rainer Vogt, Colimits, Stanley-Reisner algebras, and loop spaces, from: "Algebraic Topology: Categorical decomposition techniques", Progress in Mathematics 215, Birkhäuser (2003) 261-291 MathReview

[22] Stefan Schwede, Brooke E Shipley, Algebras and modules in monoidal model categories, Proc. London Math. Soc. 80 (2000) 491-511 MathReview

[23] Richard P Stanley, Combinatorics and Commutative Algebra, 2nd edition, Progress in Mathematics 41, Birkhäuser, Boston (1996) MathReview

[24] Dennis Sullivan, Infinitesimal computations in topology, Inst. Hautes Études Sci. Publ. Math. 47 (1977) 269-331 MathReview

[25] Rainer M Vogt, Convenient categories of topological spaces for homotopy theory, Arch. Math. 22 (1971) 545-555 MathReview

[26] Volkmar Welker, Günter M Ziegler, Rade T Živaljević, Homotopy colimits - comparison lemmas for combinatorial applications, J. Reine Angew. Math. 509 (1999) 117-149 MathReview

Department of Mathematics and Computer Science, University of Leicester

University Road, Leicester LE1 7RH, UK

and

Department of Mathematics, University of Manchester

Oxford Road, Manchester M13 9PL, UK

Email: dn8@mcs.le.ac.uk and nige@ma.man.ac.uk

Received: 21 May 2004 Revised: 23 December 2004 